Received: 23.10 .2021

Revised: 17.12 .2021

Accepted: 24.12 .2021

DOI: $10.17804 / 2410-9908.2021 .6 .068-079$

\title{
THE EFFECT OF DIRECT CURRENT IN A STEEL ROD ON THE SWITCHING FIELD DEPENDENCE OF MAGNETIC PERMEABILITY
}

\author{
V. G. Kuleev \\ M. N. Miheev Institute of Metal Physics, Ural Branch of the Russian Academy of Sciences, \\ 18 S. Kovalevskoy St., Ekaterinburg, 620108, Russian Federation \\ iD https://orcid.org/0000-0002-2122-3241 《ulejevv@imp.uran.ru \\ Corresponding author. E-mail: kulejevv@imp.uran.ru \\ Address for correspondence: ul. S. Kovalevskoy, 18, Ekaterinburg, 620108, Russian Federation
}

It is theoretically shown that in cases where the process of irreversible magnetization reversal of ferromagnetic steels is determined by displacements of only $180^{\circ}$ domain boundaries, the dependences of reversible permeability on the switching field in low-carbon steels when a direct current is passed along a rod sample should have two maxima instead of the usual one that occurs in the absence of this current. The distances between the maxima of these peaks linearly depend on the value of the constant component of the current and, consequently, on the constant circular magnetic field caused by it, perpendicular to the switching field.

This phenomenon should occur both for undeformed steel samples in the state after annealing and for samples after plastic stretching. In the latter case, elastic tensile stresses of such magnitude should act to compensate for internal residual compressive stresses. The fields of both peaks of magnetic permeability in these two cases are close to each other.

Keywords: annealed low-carbon steel, deformation, residual compressive stresses, elastic tensile stresses, EMF, circular constant and alternating magnetic fields, reversible magnetic permeability: magnetization reversal along the major hysteresis loop.

\section{Acknowledgment}

The work was performed under the state assignment of the Ministry of Education and Science of Russia (theme Diagnostics, no. AAAA-A18-118020690196-3).

\section{References}

1. Wolfart X. Vliyanie tsiklicheskikh napryazheniy [Influence of residual stresses]. In: Behavior of steels under cyclic loads, ed. by W. M. Dal, Moscow, Metallurgiya Publ., 1983, pp. 243-279. (In Russian).

2. Mishakin V.V., Mitenkov F.M., Danilova N.V., Klyushnikov V.A. The use of acoustic method to estimate the damage of 321 steel under fatigue failure. Kontrol. Diagnostika, 2012, No. 7, pp. 9-13. (In Russian).

3. Nichipuruk A.P., Stashkov A.N., Kostin V.N., Korkh M.K. Possibilities of magnetic inspection of plastic deformations preceding failures of low-carbon steels constructions. Russian Journal of Nondestructive Testing, 2009, vol. 45, No. 9, pp. 616-622. DOI: 10.1134/S1061830909090034.

4. $\quad$ Nichipuruk A.P., Rozenfeld E.V., Ogneva M.S., Stashkov A.N., Korolev A.V. An experimental method for evaluating the critical fields of moving domain boundaries in plastically tensiondeformed low-carbon wires. Russian Journal of Nondestructive Testing, 2014, vol. 50, No. 10, pp. 566-573. DOI: 10.1134/S1061830914100088. 
5. Kuleev V.G., Stashkov A.N., Tsarkova T.P., and Nichipuruk A.P. Experimental determination of critical fields of 90-degree domain wall displacement in plastically deformed lowcarbon steels. Russian Journal of Nondestructive Testing, 2018, vol. 54, No. 10, pp. 711-716. DOI: $10.1134 / \mathrm{S} 1061830918100078$.

6. Kuleev V.G., Degtyarev M.V., Stashkov A.N., and Nichipuruk A.P. On the origin of peaks of differential magnetic permeability in low-carbon steels after plastic deformation. The Physics of Metals and Metallography, 2019, vol. 120, No. 2, pp. 128-132. DOI: 10.1134/S0031918X19020108.

7. Stashkov A.N., Kuleev V.G., Nichipuruk A.P. Studying field dependence of reversible magnetic permeability in plastically deformed low-carbon steels. Russian Journal of Nondestructive Testing, 2018, vol. 54, No. 12, pp. 855-860. DOI: 10.1134/S1061830918120094.

8. Kuleev V.G., Stashkov A.N., Nichipuruk A.P. Reasons for the difference of the fields of the peaks of the reversible and differential magnetic permeability in deformed low-carbon steels. The Physics of Metals and Metallography, 2019, vol. 120, No. 7, pp. 632-638. DOI: 10.1134/S0031918X19070044.

9. Stashkov A.N., Nichipuruk A.P., Ogneva M.S., Kuleev V.G. The effect of plastic strain and the orthogonal bias field on the processes of magnetization of low-carbon steel. Russian Journal of Nondestructive Testing, 2012, vol. 48, No. 12, pp. 686-692. DOI: 10.1134/S1061830912120066.

10. Govorkov W.A., Kupalyan S.D. Teoriya elektromagnitnogo polya $v$ uprazhneniyakh $i$ zadachakh [Electromagnetic field theory in exercises and tasks]. Moscow, Vysshaya Shkola Publ., 1970, 302 p. (In Russian).

11. Gleiter G., Chalmers B. Bolsheuglovye granitsy zeren [High angle grain boundaries]. Moscow, Mir Publ., 1985, 375 p. (In Russian).

12. Kuleev V.G., Tsar'kova T.P., Sazhina E.Y. Effect of transitions of domain boundaries in plastically deformed steels on their residual magnetization. Russian Journal of Nondestructive Testing, 2016, vol. 52, No. 12, pp. 745-752. DOI: 10.1134/S1061830916120123.

13. Polivanov K.M. Teoreticheskie osnovy elektrotekhniki [Theoretical Foundations of Electrical Engineering]. Moscow, Energiya Publ, 1969, 351 p. (In Russian).

14. Bosort P. Ferromagnetizm [Ferromagnetism]. Moscow, Izd-vo inostr. lit. Publ., 1956, 784 p. (In Russian). 
Подана в журнал: 15.10 .2021

УДК 620.179.14

DOI: $10.17804 / 2410-9908.2021 .6 .068-079$

\title{
О ВЛИЯНИИ ПОСТОЯННОГО ТОКА В СТАЛЬНОМ СТЕРЖНЕ НА ЗАВИСИМОСТЬ МАГНИТНОЙ ПРОНИЦАЕМОСТИ ОТ ПЕРЕМАГНИЧИВАЮЩЕГО ПОЛЯ
}

\author{
В. Г. Кулеев \\ Институт физики металлов УрО РАН имени М. Н. Михеева, \\ 18, ул. Софьи Ковалевской, 620108, г. Екатеринбург, Российская Федерация \\ (DD https://orcid.org/0000-0002-2122-3241 @ kulejevv@imp.uran.ru
}

\author{
Ответственный автор. Электронная почта: kulejevv@imp.uran.ru \\ Адрес для переписки: ул. С. Ковалевской, 18, г. Екатеринбург, 620108, Российская Федерация
}

Теоретически показано, что в случаях, когда процесс необратимого перемагничивания ферромагнитных сталей определяется смещениями только 180-градусных доменных границ, зависимости обратимой проницаемости от перемагничивающего поля в малоуглеродистых сталях при пропускании постоянного тока вдоль стержневого образца должны иметь два максимума вместо обычного одного, имеющего место в отсутствие этого тока. Расстояния между максимумами этих пиков линейно зависят от величины постоянной составляющей тока и, следовательно, от вызванного ею постоянного циркулярного магнитного поля, перпендикулярного перемагичивающему полю.

Это явление должно иметь место как для недеформированных стальных образцов в состоянии после отжига, так и для образцов после пластического растяжения. В последнем случае должны действовать упругие растягивающие напряжения такой величины, чтобы компенсировать внутренние остаточные сжимающие напряжения. Поля обоих пиков магнитной проницаемости в этих двух случаях близки друг к другу.

Ключевые слова: отожженная малоуглеродистая сталь, деформация, остаточные сжимающие напряжения, упругие растягивающие напряжения, ЭДС, циркулярное постоянное и переменное магнитные поля, обратимая магнитная проницаемость: перемагничивание по предельной петле гистерезиса.

\section{1. Введение}

Известно, что остаточные напряжения различного происхождения [1] в стальных конструкциях определяют ресурс их безаварийной работы [2]. Поэтому их неразрушающий контроль (НК) является актуальным.

Среди разнообразных методов НК важную роль играют магнитные методы использования зависимости от внутренних напряжений $\sigma_{i}$ различных магнитных параметров.

В настоящей работе рассмотрена обратимая магнитная проницаемость $\mu_{\mathrm{rev}}(\mathrm{H})$, где $\mathrm{H}-$ внутреннее магнитное поле. Кроме него еще действуют дипольные поля от имеющихся внутри стали магнитных неоднородностей, которыми мы в дальнейшем пренебрегаем. Эксперименты в этой области НК можно разделить на две группы: в первой группе переменное магнитное поле $\mathrm{h}(\mathrm{t})$ параллельно перемагничивающему полю Н [3]; во второй - это поле перпендикулярно: $\mathbf{h} \perp \mathbf{H}$ [4]. В последнем случае это поле создается обычно путем пропускания тока і вдоль стального образца; причем в общем случае этот ток может иметь и переменную, и постоянную составляющие: 


$$
\mathrm{i}=\mathrm{i}(\mathrm{t})+\mathrm{i}_{0},
$$

которые создают соответствующие циркулярные магнитные поля (в цилиндрической системе координат):

$$
\mathrm{h}_{\varphi}=\mathrm{h}_{\varphi}(\mathrm{r}, \mathrm{t})+\mathrm{h}_{\varphi}{ }^{0}
$$

Заметим, что учет постоянной составляющей тока может оказаться важным при непосредственном контроле остаточных напряжений в стальных конструкциях, по которым по тем или иным причинам течет паразитный постоянный ток.

В [5] предложен способ выделения вклада смещений 90-градусных ДГ в магнитную проницаемость. Для этого должен быть выделен вклад в проницаемость и от смещений 180градусных ДГ. В [5], как и в следующих [6-8], постоянное и переменное поля были параллельны. Настоящая работа относится к случаю, когда эти поля перпендикулярны.

Цель работы - исследование зависимости проницаемости $\mu_{\mathrm{rev}}(\mathrm{H})$ в условиях, когда циркулярное поле $\mathrm{h}_{\varphi}$ имеет помимо переменной еще и постоянную составляющую $\mathrm{h}_{\varphi}{ }^{0}$ и когда намагниченность может изменяться с помощью смещений только 180-градусных ДГ. Это непосредственно относится к отожженным образцам, где имеют место напряжения только второго рода $\sigma_{\mathrm{i}}^{(2)}$.

Также рассмотрены и деформированные стальные образцы в условиях, когда к ним прилагаются упругие растягивающие напряжения такой величины, чтобы скомпенсировать внутренние остаточные сжимающие напряжения [5-8]: в этом случае также возможны смещения только 180-градусных ДГ [4].

\section{2. Поля пиков $\mu_{\mathrm{rev}}{ }^{180}(\mathrm{H})$ в отдельных зернах поликристалла}

Рассмотрим необратимые 180-градусные смещения в стержнях из малоуглеродистой отожженной стали в самом простом случае: $\mathrm{h}_{\varphi}{ }^{0}=0 ; \mathrm{h}_{\varphi}(\mathrm{t}) \neq 0$ при изменении намагниченности $\mathrm{M}(\mathrm{H})$ по спинке петли гистерезиса с уменьшением поля от насыщения. Нас будет интересовать область полей, где происходят необратимые смещения ДГ: от $+40 \mathrm{~A} / \mathrm{cm}$ до -40 A/cм [5-8] и находятся все пики магнитной проницаемости, являющиеся параметрами контроля остаточных напряжений [5-8].

В силу малости магнитоупругой энергии и энергии во внутреннем поле $\mathrm{H}$, по сравнению с энергией магнитной анизотропии в железе [4, 5, 7], все магнитные моменты зерен находятся в своих легких направлениях типа [100], которые наиболее близки к полю, т. е., в исходном конусе с углами и от $\left(+55^{\circ}\right)$ до $-\left(55^{\circ}\right)$ (рис. 1$)$. И эти направления моментов сохраняются вплоть до их необратимых смещений на $180^{\circ}$ в другие легкие направления: $(\theta+$ $180^{\circ}$ ). Такие смещения должны происходить, когда разность энергий начального $\mathrm{E}_{1}(\theta)$ и конечного состояний $\mathrm{E}_{2}\left(\theta+180^{\circ}\right)$ становится равной (или несколько больше) энергии потенциального барьера 180-градусной ДГ $\left(\mathrm{M}_{\mathrm{S}} \mathrm{H}_{\mathrm{b}}{ }^{180}\right)$ :

$$
\begin{gathered}
\mathrm{E}_{1}(\theta)=-\mathrm{H}_{0} \mathrm{M}_{\mathrm{S}} \cos \theta \\
\mathrm{E}_{2}\left(\theta+180^{\circ}\right)=+\mathrm{H}_{0} \mathrm{M}_{\mathrm{S}} \cos \theta ; \\
\Delta \mathrm{E}(\theta)=-2 \mathrm{H}_{0} \mathrm{M}_{\mathrm{S}} \cos \theta=\mathrm{M}_{\mathrm{S}} \mathrm{H}_{\mathrm{b}}{ }^{180} ; \\
\mathrm{H}_{\mathrm{\kappa р}}{ }^{180}(\theta)=-\mathrm{H}_{\mathrm{b}}{ }^{180} /(2 \cos \theta) ; \\
\mathrm{H}_{\mathrm{\kappa р}}{ }^{180}\left(55^{\circ}\right)=-0,872 \mathrm{H}_{\mathrm{b}}{ }^{180} .
\end{gathered}
$$

Наибольшее значение этого поля для отдельного зерна имеет место при $\theta=55^{\circ}$. 
Рассмотрим далее влияние постоянного циркулярного поля $\mathrm{h}_{\varphi}{ }^{0}$, вызванного постоянным током через образец, на зависимости магнитной проницаемости в отдельном зерне, находящемся в составе поликристалла. Оно добавляет свою энергию $\mathrm{E}_{\varphi}$, влияющую на магнитные моменты зерен $\mathrm{M}(\theta)$ :

$$
\mathrm{E}_{\varphi}(\theta)=-\mathrm{M}_{\mathrm{S}} \mathrm{h}_{\varphi}{ }^{0} \cos (\theta \pm 90)= \pm \mathrm{M}_{\mathrm{S}} \mathrm{h}_{\varphi}{ }^{0} \sin \theta
$$

Эта энергия имеет разный знак в зависимости от знака и. При поле $\mathrm{h}_{\varphi}^{0}$, направленном вверх, знак $(+)$ относится к половине зерен в исходном конусе с $0 \leq \theta \leq 55^{\circ}$; а знак (-) относится ко второй половине зерен, у которых $0 \geq \theta \geq-55^{\circ}$. Такое распределение справедливо для любого сечения единичной сферы, изображенного на рис. 1. При поле $\mathrm{h}_{\varphi}{ }^{0}$, направленном вниз, поля (4) просто поменяются местами.

Выражение (4) свидетельствует о том, что на магнитный момент любого зерна в исходном конусе одновременно действуют два разных постоянных поля: одно от перемагничивающего внутреннего поля Н согласно (3), а другое - от постоянного циркулярного поля $\mathrm{h}_{\varphi}{ }^{0}$ согласно (4), так что поля, действующие на зерна с $\theta>0$ и с $\theta<0$, имеют вид:

$$
\begin{aligned}
& \mathrm{H}_{1}{ }^{180}(\theta)=-\mathrm{H}_{0} \cos \theta+\mathrm{h}_{\varphi}{ }^{0} \sin \theta ; \\
& \mathrm{H}_{2}{ }^{180}(\theta)=-\mathrm{H}_{0} \cos \theta-\mathrm{h}_{\varphi}{ }^{0} \sin \theta .
\end{aligned}
$$

После 180-градусного смещения на эти моменты $\mathrm{M}(\theta)$ действуют поля:

$$
\begin{aligned}
& \mathrm{H}_{1}{ }^{180}\left(\theta+180^{\circ}\right)=\mathrm{H}_{0} \cos \theta-\mathrm{h}_{\varphi}{ }^{0} \sin \theta ; \\
& \mathrm{H}_{2}{ }^{180}\left(\theta+180^{\circ}\right)=\mathrm{H}_{0} \cos \theta+\mathrm{h}_{\varphi}{ }^{0} \sin \theta .
\end{aligned}
$$

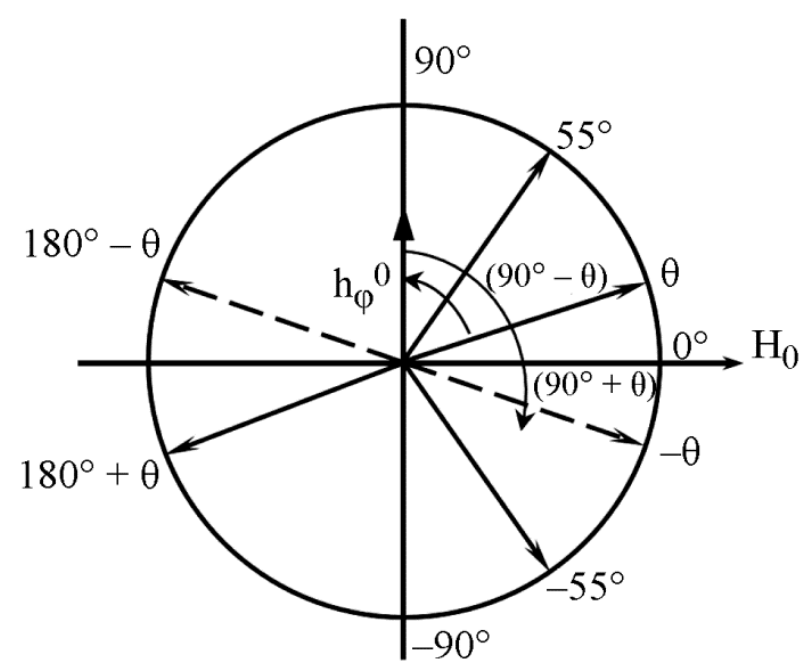

Рис. 1. Схема 180-градусных переходов в образцах малоуглеродистой стали: сплошные линии - переходы из области, где $\theta>0$; штриховые линии - переходы из области исходного конуса, где $\theta<0$

180-градусные необратимые смещения в указанных выше двух половинах зерен поли-

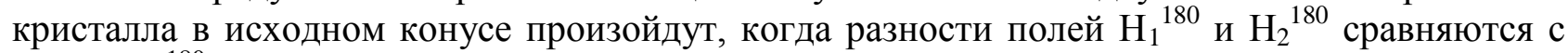
полем $\mathrm{H}_{5}{ }^{180}$. В результате найдем поля пиков магнитной проницаемости для каждой из половин зерен исходного конуса (рис. 1):

$$
\mathrm{H}_{\text {пр }}(\theta)=-\mathrm{H}_{\mathrm{b}}^{180} /(2 \cos \theta)+\mathrm{h}_{\varphi}{ }^{0} \operatorname{tg} \theta
$$




$$
\mathrm{H}_{\text {лев }}(\theta)=-\mathrm{H}_{\mathrm{5}}{ }^{180} /(2 \cos \theta)-\mathrm{h}_{\varphi}{ }^{0} \operatorname{tg} \theta .
$$

В итоге вместо стандартного при $\mathrm{h}_{\varphi}{ }^{0}=0$ одного пика проницаемости при $\mathrm{h}_{\varphi}{ }^{0} \neq 0$ [7]) в поликристалле должно иметь место два пика магнитной проницаемости $\mu_{\text {rev }}(\mathrm{H}, \theta)$. Причем правый пик, в отличие от (3), может появиться в области положительных полей $\mathrm{H}_{0}>0$, если выполняется неравенство:

$$
\mathrm{h}_{\varphi}{ }^{0} \sin \theta>\mathrm{H}_{\mathrm{b}}{ }^{180} / 2
$$

Заметим, что это соотношение можно использовать как независимый способ определения величины ПБ для 180-градусных ДГ. Удвоение числа пиков в перпендикулярных полях было обнаружено ранее в экспериментальной работе [9], где исходя из размагниченного состояния при увеличении перемагничивающего поля было обнаружено появление второго пика кривой $\mu(\mathrm{H})$ при включении перпендикулярного поля. При выключении этого поля имел место только один пик. Такое поведение обратимой проницаемости в [9] не нашло физического объяснения.

Заметим, что вышеприведенные формулы (3)-(8) относятся к отдельным зернам поликристалла без учета особенностей их распределения в исходном конусе (рис. 1).

\section{3. Влияние особенностей распределения зерен в стальном поликристалле на обратимую магнитную проницаемость}

С учетом, что на опыте измеряется зависимость ЭДС от поля Н с помощью катушки, намотанной на образец, она пропорциональна обратимой магнитной проницаемос ти. Поскольку нас интересуют только пики $\mu_{\mathrm{rev}}{ }^{180}\left(\mathrm{H}_{0}\right)$, обусловленные 180 -градусными смещениями, то далее следует учесть еще ряд факторов, влияющих на величины измеряемой ЭДС и соответственно, обратимой магнитной проницаемости.

К ним относятся следующие факторы.

1. Учет роли изменения величин полей $\mathrm{h}_{\varphi}{ }^{0}$ и $\mathrm{h}_{\varphi}(\mathrm{r}, \mathrm{t}) \mathrm{c}$ ростом радиуса стержня от нуля и до $\mathrm{r}_{0}$ (последнего с учетом скин-эффекта).

Согласно [10] зависимости полей $\mathrm{h}_{\varphi}(\mathrm{r}, \mathrm{t})$ и $\mathrm{h}_{\varphi}{ }^{0}$ от радиуса стержня имеют вид:

$$
\begin{gathered}
\mathrm{h}_{\varphi}^{0}(\mathrm{r})=\left(\mathrm{i}_{0} \mathrm{r}\right) /\left[2 \pi\left(\mathrm{r}_{0}\right)^{2}\right] ; \\
\mathrm{h}_{\varphi}^{0}(\mathrm{r}, \mathrm{t})=\mathrm{h}_{\varphi} \exp (-\mathrm{r} / \delta) \cos (\omega \mathrm{t}-\mathrm{r} / \delta) .
\end{gathered}
$$

Здесь $\delta$ - глубина скин-слоя; $\omega$ - круговая частота; $\mathrm{r}_{0}$ - радиус стержня; $\mathrm{r}$ - его текущее значение.

При данном значении и поле $\mathrm{h}_{\varphi}{ }^{0}\left(\mathrm{r}_{0}\right)$ является максимальным. С увеличением радиуса стержня $\mathrm{r}$ от нуля и до $\mathrm{r}_{0}$ квадратично $\mathrm{c} r$ растут объемы его элементарных слоев, достигая максимума при $r=r_{0}$ и пропорционально увеличивая тем самым величину ЭДС а значит и $\mu_{\mathrm{rev}}{ }^{180}(\mathrm{H})$. Существенно, что слои стержня $\mathrm{c} \mathrm{r}<\mathrm{r}_{0}$ будут давать меньшие значения ЭДС (или $\left.\mu_{\text {rev }}\right)$, чем при $r=r_{0}$, и их роль, следовательно, сводится только к уширению регистрируемого максимума $\mu_{\text {rev }}^{180}(\mathrm{H})$. В итоге максимум из-за его зависимости от $r(9)$ определяется полем на поверхности стержня.

2. При увеличении угла и от 0 до $55^{\circ}$ в исходном конусе (рис. 1) число зерен $\mathrm{N}(\theta)$ в элементарном слое растет с ростом $\mathrm{r}$ в соответствии с известным законом геометрии: $\mathrm{r} 2 \pi(1-\cos \theta)$, справедливым для единичной сферы:

$$
\mathrm{N}(\theta)=\mathrm{A} 2 \pi(1-\cos \theta)
$$


где А - константа. Так как ЭДС пропорциональна числу зерен, в которых происходят 180градусные скачки, то в выражении для ЭДС должен быть включен также множитель (10).

3. Учет обстоятельства, что магнитные моменты зерен в исходном конусе направлены под углом и к полю $\mathbf{H}_{\mathbf{0}}$ и под углами $(90 \pm \theta)$ к полю $\mathbf{h}_{\boldsymbol{\varphi}}^{\mathbf{0}}$, а ось измерительной катушки параллельна стальному стержню и полю $\mathbf{H}_{\mathbf{0}}$. В этом случае, как известно [10], исходные выражения для полей пиков (7) отдельных зерен с данным значением угла и должны быть умножены на $[\cos \theta]$.

4. Учет вклада пар зерен с большеугловыми границами (БУГ) в связи с квадратичной зависимостью их числа от разности углов $\Delta \theta$ между осями [100] в парах зерен с БУГ [5-8].

\section{4. Отожженные стальные образцы}

Рассмотрим стальные образцы в отожженном состоянии, когда в них действуют остаточные напряжения только второго рода $\sigma_{\mathrm{i}}^{(2)}$, которые уравновешены внутри каждого зерна [1]; в этом случае все необратимые смещения ДГ являются 180-градусными. Учтем далее, что все зерна поликристалла делятся на две подгруппы: зерна с малоугловыми границами (МУГ) и зерна с БУГ [11]. Их, как показано в [12], на основе анализа диаграмм парных соседств зерен поликристалла соответственно 55,2 \% и 44,8 \% от всего числа зерен в поликристалле $\mathrm{N}_{0}$. И хотя магнитные моменты зерен в обеих подгруппах ориентированы в исходном конусе хаотично, пары зерен с БУГ отличаются от зерен с МУГ тем, что их число растет квадратично с уменьшением величины $\Delta \theta$ - угла между осями [100] двух соседних зерен с БУГ (рис. 1). При этом $\Delta \theta=\theta_{2}-\theta_{1}$, где $\theta_{2}$ и $\theta_{1}-$ углы между осями [100] зерен 1 и 2 , наиболее близкими к полю, и полем $\mathbf{H}_{\mathbf{0}}$. Отмеченный квадратичный рост числа зерен с БУГ про-

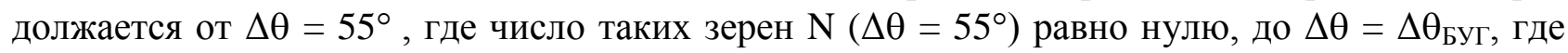

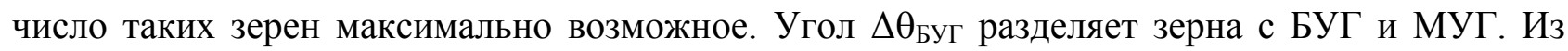
диаграммы парных соседств получено [11], что для ОЦК-решетки железа $\Delta \theta_{\text {Буг }}=18,33^{\circ}$.

В итоге при данном значении $\Delta \theta$ число зерен с БУГ равно [12]:

$$
\mathrm{N}_{\text {Буг }}(\Delta \theta)=\mathrm{A}_{1}\left(55^{\circ}-\Delta \theta\right)^{2} .
$$

Здесь $\mathrm{A}_{1}$ - постоянный множитель. Максимальное значение этого числа равно $\mathrm{N}_{\text {БуГ }}\left(18,33^{\circ}\right)=1344 \mathrm{~A}_{1}$.

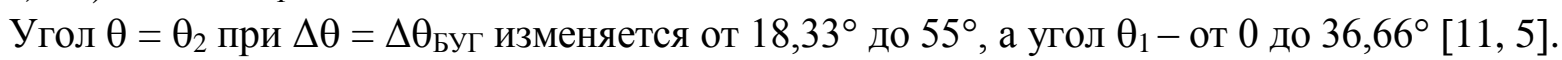
В результате того, что при $\theta=55^{\circ}$ длина окружности вокруг исходного конуса максимальна, то в случае зерен с БУГ величина ЭДС должна достигать своего максимума также при $\theta=55^{\circ}$, как и для зерен с МУГ.

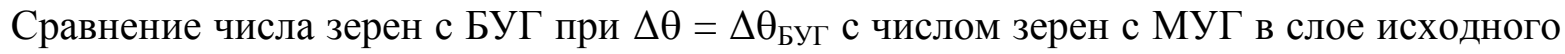
конуса при $\theta=55^{\circ}$ показывает, что главный вклад в пик ЭДС (или, что то же самое, в пропорциональный ему пик обратимой магнитной проницаемости $\mu_{\mathrm{rev}}\left(\mathrm{H}_{0}\right)$ дают зерна с БУГ: их число больше в 1,3 раза числа зерен с МУГ при $\theta=55^{\circ}$.

В результате учета всего сказанного общие выражения для полей правого и левого пиков ЭДС при $\mathrm{h}_{\varphi}{ }^{0} \neq 0$ для зерен с любыми и в интервалах: $0 \div 55^{\circ}$ (для поля $\mathrm{H}_{\text {пр }}{ }^{180}$ ) и $0 \div-55^{\circ}$ (для поля $\mathrm{H}_{\text {лев }}{ }^{180}$ ) имеют вид:

$$
\begin{aligned}
& \mathrm{H}_{\text {пр }}{ }^{180}\left(\theta, \mathrm{h}_{\varphi}{ }^{0}\right)=-\pi(1-\cos \theta) \mathrm{H}_{\mathrm{Б}}{ }^{180}+2 \pi(1-\cos \theta) \times \mathrm{h}_{\varphi}{ }^{0} \sin \theta \\
& \mathrm{H}_{\text {лев }}{ }^{180}\left(\theta, \mathrm{h}_{\varphi}{ }^{0}\right)=-\pi(1-\cos \theta) \mathrm{H}_{\mathrm{Б}}{ }^{180}-2 \pi(1-\cos \theta) \times \mathrm{h}_{\varphi}{ }^{0} \sin \theta .
\end{aligned}
$$

Поля двух наибольших пиков обратимой магнитной проницаемости должны иметь место при $\theta=55^{\circ}\left(\mathrm{H}_{\text {пр }}{ }^{180}\right)$ и при $\theta=-55^{\circ}\left(\mathrm{H}_{\text {лев }}{ }^{180}\right)$, где: 


$$
\begin{aligned}
& \mathrm{H}_{\text {пр }}{ }^{180}\left(55^{\circ}, \mathrm{h}_{\varphi}{ }^{0}\right)=-1,34 \mathrm{H}_{\mathrm{Б}}{ }^{180}+2,19 \mathrm{~h}_{\varphi}{ }^{0} ; \\
& \mathrm{H}_{\text {лев }}{ }^{180}\left(55^{\circ}, \mathrm{h}_{\varphi}{ }^{0}\right)=-1,34 \mathrm{H}_{\mathrm{Б}}{ }^{180}-2,19 \mathrm{~h}_{\varphi}{ }^{0} .
\end{aligned}
$$

Перейдем к анализу выражений (12), справедливых для зерен с любыми значениями угла и из указанных выше интервалов его изменения в исходном конусе (рис. 1).

Полусумма этих выражений дает удвоенное «невозмущенное» значение поля пиков 180-градусных смещений, которое отличается от (3) в силу учета особенностей распределения зерен в поликристалле (10) и ориентации измерительной катушки: в нашем случае «невозмущенное» значение этого поля при $\theta=55^{\circ}$ есть $(13)$ :

$$
\mathrm{H}_{\text {кр }}{ }^{180}\left(55^{\circ}\right)=-1,34 \mathrm{H}_{\mathrm{b}}{ }^{180} \text {. }
$$

Разность выражений (13) $\Delta \mathrm{H}^{180}$ дает расстояние между двумя пиками ЭДС. Оно линейно зависит от величины $\mathrm{h}_{\varphi}{ }^{0}$ :

$$
\Delta \mathrm{H}^{180}\left(55^{\circ}\right)=4,38 \times \mathrm{h}_{\varphi}^{0} .
$$

Сравнение с опытом общего соотношения для $\Delta \mathrm{H}^{180}$, полученного из (12)

$$
\Delta \mathrm{H}^{180}(\theta)=4 \pi \mathrm{h}_{\varphi}{ }^{0} \sin \theta(1-\cos \theta),
$$

и знание величины $\mathrm{h}_{\varphi}{ }^{0}$ позволят оценить характер зависимости второго члена в (12) от $\theta$.

Из (13) легко найти значение $\mathrm{H}_{\mathrm{b}}{ }^{180}$ по величине $\mathrm{h}_{\varphi}{ }^{0}$. При $\theta=55^{\circ}$ оно равно:

$$
\mathrm{H}_{\mathrm{b}}{ }^{180}=1,638 \mathrm{~h}_{\varphi}^{0} .
$$

Это же значение получается и из (8) при $\theta=55^{\circ}$.

\section{5. Ширины 180-градусных пиков при изменении величины постоянного циркулярного поля $\mathbf{h}_{\varphi}{ }^{0}$}

Рассмотрим механизмы уширения двух пиков зависимости $\mu_{\mathrm{rev}}\left(\mathrm{H}_{0}\right)$ при $\mathrm{h}_{\varphi}{ }^{0} \neq 0$.

Необходимо различать «естественную ширину» локальных пиков $\delta \mathrm{h}(\mathrm{r}, \theta)$, имеющую место для каждого зерна с данным значением угла и внутри исходного конуса, от интегральной ширины каждого из двух пиков (12), обусловленной всеми зернами поликристалла из каждой половины исходного конуса, т. е., из областей $\theta>0$ и $\theta<0$ (рис. 1).

Эта «естественная ширина линии» $\delta \mathrm{h}(\mathrm{r}, \theta)$ определяется зависимостью модуля переменного циркулярного поля $\mathrm{h}_{\varphi}(\mathrm{r}, \mathrm{t})$ от радиуса стержня. Величина $\delta \mathrm{h}(\mathrm{r}, \theta)$ определяет амплитуду локального пика для каждого зерна с данными значениями $\mathrm{r}$ и $\theta$ при данной амплитуде переменного поля.

Рассмотрим механизмы формирования локальных пиков, определяющих их ширины $\delta \mathrm{h}(\mathrm{r}, \theta)$ и, следовательно, их амплитуды. Один из этих механизмов отмечен выше. Он обусловлен зависимостью числа зерен от радиуса стального стержня согласно (9) и, следовательно, одинаков для обоих членов в (12): для члена, пропорционального $\mathrm{H}_{5}{ }^{180}$, и для члена, пропорционального $\mathrm{h}_{\varphi}{ }^{0}$. Этот механизм вместе с механизмом поглощения энергии переменного поля (из-за скин-эффекта) [9] определяет естественную ширину каждого локального пика данного зерна, как и его амплитуду.

В первом приближении форма любого локального пика похожа на обычную резонансную $[13,14]$, хотя механизмы ее образования здесь другие. Для дальнейшего важно, что 
с ростом амплитуды пика (например при увеличении $\mathrm{h}(\mathrm{r}, \mathrm{t}))$ в том же отношении должна возрастать и ширина локального пика $\delta \mathrm{h}(\mathrm{r}, \mathrm{t})$.

Так как каждому значению угла $\theta>0$ соответствует точно такое же по модулю значение $\theta<0$ (рис. 1), при каждом $|\theta|$ будем иметь два локальных пика в соответствии с (12) в строго определенных полях и обладающих соответствующей шириной.

Если теперь просуммировать все локальные пики в обоих диапазонах изменения угла и $\left(0<\theta<55^{\circ}\right.$ и $\left.0>\theta>-55^{\circ}\right)$, то получим общую кривую изменения обратимой проницаемости от угла $\theta$ (12), содержащую два пика. Так как каждому значению угла и соответствует свое значение поля согласно (12), то легко пересчитать полученные таким образом ширины обоих пиков в соответствующие их полевые значения $\Delta \mathrm{H}_{\text {пр }}$ и $\Delta \mathrm{H}_{\text {лев }}$ при изменении и от $0^{\circ}$ до $\pm 55^{\circ}$. Эти интегральные ширины определяют и амплитуды интегральных пиков кривой $\mu_{\text {rev }}\left(\mathrm{H}_{0}\right)$, обусловленных отмеченными выше вкладами зерен из двух половин исходного конуса (рис. 1). Из-за того, что числа зерен в двух половинах исходного конуса одинаковы, площади двух пиков должны быть также одинаковыми, что будет влиять на относительную форму этих двух интегральных пиков следующим образом.

В области малых $\mathrm{h}_{\varphi}{ }^{0}$, когда члены в (12), пропорциональные $\mathrm{h}_{\varphi}{ }^{0}$, меньше членов, пропорциональных $\mathrm{H}_{\mathrm{b}}{ }^{180}$, главный вклад в общую ширину обоих пиков $\mu_{\mathrm{rev}}\left(\mathrm{H}_{0}\right)$ должен давать член с $\mathrm{H}_{5}{ }^{180}$. Так как в правом пике (поле $\mathrm{H}_{\text {пр }}{ }^{180}$ ) вклады вычитаются, то этот пик должен быть более узким и, следовательно, более высоким, чем левый пик (поле $\mathrm{H}_{\text {лев }}{ }^{180}$ ), где вклады двух членов в (12) складываются, в результате чего ширина этого пика должна равняться сумме «естественных ширин». В результате этот пик должен быть более широким и вместе с тем более низким. Причиной такого поведения является требование одинаковости площадей обоих пиков.

В случае когда член $\mathrm{c} \mathrm{h}_{\varphi}{ }^{0}$ заметно превышает величину члена с $\mathrm{H}_{5}{ }^{180}$ в (12), он должен давать главный вклад в ширину обоих пиков $\left(\Delta \mathrm{H}_{\text {пр и }} \Delta \mathrm{H}_{\text {лев}}\right)$, в результате чего пики должны сближаться и по ширине, и по высоте, хотя правый пик все же должен быть несколько выше левого за счет малого члена с $\mathrm{H}_{\mathrm{b}}{ }^{180}$. На рис. 2 представлена схема изменения обратимой проницаемости с ростом постоянного циркулярного поля. Кроме того, наши выводы интересно проверить на опыте.

\section{6. Деформированные стальные образцы при упругом растяжении}

Известно [5-8], что в деформированных растяжением стальных образцах существуют остаточные сжимающие напряжения первого рода $\sigma_{i}^{(1)}[1]$, ориентированные вдоль действовавшей ранее нагрузки (и поля $\mathbf{H}_{\mathbf{0}}$ ). Эти напряжения являются причиной появления необратимых смещений 90-градусных ДГ при изменении намагниченности по спинке петли гистерезиса [5-8]. В обратимой магнитной проницаемости $\mu_{\text {rev }}\left(\mathrm{H}_{0}\right)$ такие смещения вызывают два пика: при $\mathrm{H}_{0}>0$ и при $\mathrm{H}_{0}<0[7,8]$.

В итоге общее необратимое изменение проницаемости с полем определяется суммой 90-градусных и 180-градусных переходов. Поэтому, чтобы получить только 180-градусные переходы при перемагничивании деформированных образцов, нужно приложить к ним упругое растягивающее напряжение $\sigma_{0}>0$ такой величины, которая достаточна для компенсации всех остаточных сжимающих напряжений первого рода $\sigma_{i}^{(1)}$, существующих только в зернах с БУГ [5-8, 12].

Напряжения $\sigma_{i}^{(1)}$, как известно $[5,11]$, изменяются от своего минимального значения

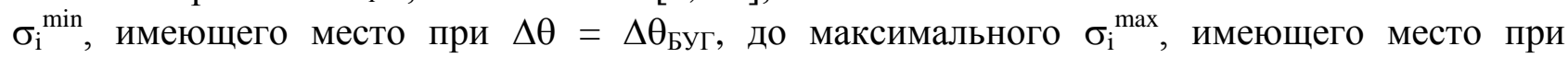
$\Delta \theta=55^{\circ}$. В результате полная компенсация всех остаточных сжимающих напряжений наступит при $\sigma_{0}=\left|\sigma_{\mathrm{i}}{ }^{\mathrm{max}}\right|$. 


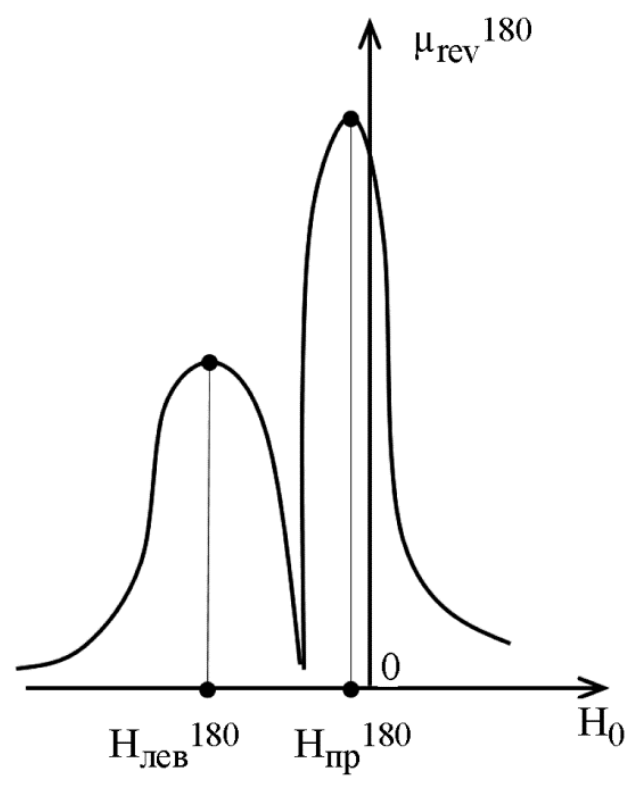

$a$

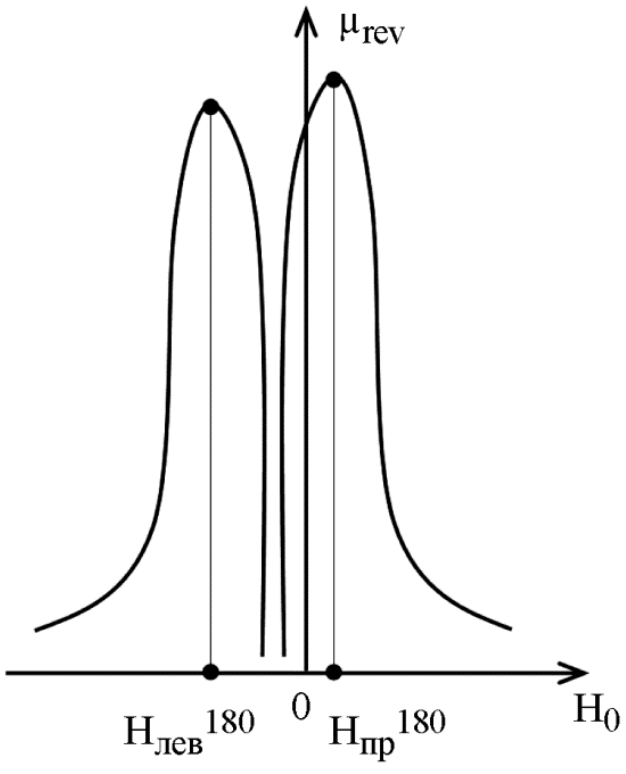

$\sigma$

Рис. 2. Схема изменения вида кривой $\mu_{\mathrm{rev}}{ }^{180}(\mathrm{H})$ (12) с ростом величины постоянного циркулярного поля $\mathrm{h}_{\varphi}{ }^{0}: a$ - член в с $\mathrm{H}_{\mathrm{b}}{ }^{180}$ больше члена $\mathrm{c} \mathrm{h}_{\varphi}{ }^{0}$; $\sigma$ - член с $\mathrm{H}_{\mathrm{b}}{ }^{180}$ значительно меньше члена $\mathrm{c}_{\varphi}{ }^{0}$

В частности, при деформации $\varepsilon=8,4 \%$ отожженной стали Ст3 согласно $[5,6]$ $\sigma_{\mathrm{i}}^{\max }=-300$ МПа. В этом случае при $\sigma_{0}=+300$ МПа все остаточно сжатые зерна (зерна с БУГ) будут находиться в растянутом состоянии, не говоря уже о зернах с МУГ, в которых остаточные напряжения первого рода отсутствуют.

В итоге все зерна стального образца будут находиться в растянутом состоянии, и при перемагничивании деформированного и растянутого упруго стального образца имеют место только необратимые смещения180-градусных ДГ [13], ситуация с которыми полностью совпадает с таковой в рассмотренном ранее случае отожженных образцов.

Будет только сравнительно небольшое различие этих случаев, обусловленное некоторым увеличением эффективной константы анизотропии по сравнению с отожженным состоянием, где она равнялась $\mathrm{K}_{1}$ [14]. Новая эффективная константа анизотропии при упругом растяжении К ${ }_{э ф}$ больше, чем К $_{1}$, и в первом приближении [14] равна:

$$
\mathrm{K}_{\ni \phi}=\mathrm{K}_{1}+1,5 \lambda_{100} \cdot \sigma_{0} .
$$

В приведенном в качестве примера случае ( $\varepsilon=8,4 \% ; \sigma_{0}=300$ МПа; Ст3) второй член в (18) составляет $20 \%$ от первого. Согласно [15] это приводит к небольшому сужению 180градусной ДГ (максимально до $8 \%$ ), в результате чего также незначительно возрастет поле потенциального барьера 180 -градусных $\Delta \theta \mathrm{H}_{\mathrm{b}}{ }^{180}$ (примерно на $4 \%$ ). В остальном случай деформированных и затем растянутых упруго стальных образцов полностью совпадает с рассмотренным ранее случаем отожженных образцов.

\section{7. Выводы}

1. Показано, что циркулярное постоянное магнитное поле при перемагничивании стали должно приводить к двум максимумам зависимости обратимой магнитной проницаемости $\mu_{\text {rev }}\left(\mathrm{H}_{0}\right)$ и в отожженном, и в деформированном состояниях. В последнем случае должны накладываться упругие растягивающие напряжения такой величины, чтобы были возможны только 180-градусные смещения. 
2. Установлено, что расстояния между полями максимумов $\mathrm{H}_{\text {пр }}{ }^{180}$ и $\mathrm{H}_{\text {лев}}{ }^{180}$ на полевых зависимостях обратимой магнитной проницаемости должны линейно зависеть от величины постоянной составляющей циркулярного магнитного поля $\mathrm{h}_{\varphi}{ }^{0}$ на поверхности стального стержня.

3. Предсказан характер изменения относительной формы двух пиков кривой $\mu_{\text {rev }}{ }^{180}\left(\mathrm{H}_{0}\right)$ с ростом величины постоянного циркулярного поля $\mathrm{h}_{\varphi}^{0}$, определяемый, во-первых, равенством числа зерен, участвующих в создании двух пиков, и, во-вторых, относительной величиной двух вкладов в поля пиков: вклада, пропорционального полю потенциального барьера для 180-градусных смещений $\mathrm{H}_{5}{ }^{180}$, и вкладу, пропорциональному постоянному циркулярному полю $\mathrm{h}_{\varphi}{ }^{0}$ на поверхности стержня.

4. В работе также показано, что главный вклад в поля обоих пиков зависимости $\mu_{\mathrm{rev}}{ }^{180}(\mathrm{H})$ дают зерна с БУГ, что обусловлено квадратичной зависимостью их числа от $\Delta \theta$ - разности углов между осями [100] любых двух граничащих друг с другом зерен с БУГ.

5. Из материала работы следует, что постоянные токи, которые могут протекать по контролируемым стальным изделиям, неизбежно искажают форму полевых зависимостей обратимой магнитной проницаемости (по сравнению, например, с результатами работ $[7,8]$ ), что необходимо учитывать при определении величин остаточных механических напряжений.

\section{Благодарность}

Работа выполнена в рамках государственного задания МИНОБРНАУКИ России (тема «Диагностика», № АAАA-A18-118020690196-3).

\section{Литература}

1. Вольфарт Х. Влияние остаточных напряжений // Поведение сталей при циклических нагрузках / под ред. В. М. Даля. - М. : Металлургия, 1983. - С. 243-279.

2. Использование акустического метода оценки поврежденности стали при усталостном разрушении / В. В. Мишакин, Ф. М. Митенков, Н. В. Данилова, В. А. Клюшников. Контроль. Диагностика. - 2012. - № 7. - С. 9-13.

3. Possibilities of magnetic inspection of plastic deformations preceding failures of low-carbon steels constructions / A. P. Nichipuruk, A. N. Stashkov, V. N. Kostin, M. K. Korkh // Russian Journal of Nondestructive Testing. - 2009. - Vol. 45, No. 9. - P. 616-622. - DOI: 10.1134/S1061830909090034.

4. An experimental method for evaluating the critical fields of moving domain boundaries in plastically tension-deformed low-carbon wires / A. P. Nichipuruk, E. V. Rozenfeld, M. S. Ogneva, A. N. Stashkov, A. V. Korolev // Russian Journal of Nondestructive Testing. - 2014. - Vol. 50, No. 10. - P. 566-573. - DOI: 10.1134/S1061830914100088.

5. Experimental determination of critical fields of 90-degree domain wall displacement in plastically deformed low-carbon steels / V. G. Kuleev, A. N. Stashkov, T. P. Tsarkova, and A. P. Nichipuruk // Russian Journal of Nondestructive Testing. - 2018. - Vol. 54, No. 10. - P. 711-716. DOI: $10.1134 / \mathrm{S} 1061830918100078$.

6. On the origin of peaks of differential magnetic permeability in low-carbon steels after plastic deformation / V. G. Kuleev, M. V. Degtyarev, A. N. Stashkov, and A. P. Nichipuruk // The Physics of Metals and Metallography. - 2019. - Vol. 120, No. 2. - P. 128-132. - DOI: 10.1134/S0031918X19020108.

7. Stashkov A. N., Kuleev V. G., Nichipuruk A. P. Studying field dependence of reversible magnetic permeability in plastically deformed low-carbon steels // Russian Journal of Nondestructive Testing. - 2018. - Vol. 54, No. 12. - P. 855-860. - DOI: 10.1134/S1061830918120094.

8. Kuleev V. G., Stashkov A. N., Nichipuruk A. P. Reasons for the difference of the fields of the peaks of the reversible and differential magnetic permeability in deformed low-carbon steels // The Physics of Metals and Metallography. - 2019. - Vol. 120, No. 7. - P. 632-638. DOI: $10.1134 / \mathrm{S} 0031918 X 19070044$. 
9. The effect of plastic strain and the orthogonal bias field on the processes of magnetization of low-carbon steel / A. N. Stashkov, A. P. Nichipuruk, M. S. Ogneva, V. G. Kuleev // Russian Journal of Nondestructive Testing. - 2012. - Vol. 48, No. 12. - P. 686-692. - DOI: 10.1134/S1061830912120066.

10. Говорков В. А., Купалян С. Д. Теория электромагнитного поля в упражнениях и задачах. - М. : Высшая школа, 1970. - 302 с.

11. Глейтер Г., Чалмерс Б. Большеугловые границы зерен. - М. : Мир, 1985. - 375 с.

12. Kuleev V. G., Tsar'kova T. P., Sazhina E. Y. Effect of transitions of domain boundaries in plastically deformed steels on their residual magnetization // Russian Journal of Nondestructive Testing. - 2016. - Vol. 52, No. 12. - P. 745-752. - DOI: 10.1134/S1061830916120123.

13. Поливанов К. М. Теоретические основы электротехники. - М. : Энергия, 1969. - 351 с.

14. Бозорт Р. Ферромагнетизм / пер. с англ. - М. : Изд-во иностр. лит., 1956. - 784 с. 